\title{
Increasing Learner's Level of Motivation in Mathematics Education Through the Use of Uncompleted Situations
}

\author{
M. Rodionov \\ Penza State Pedagogical University, \\ Penza, Russia
}

\author{
Z. Dedovets \\ The University of the West Indies, \\ Trinidad and Tobago
}

\begin{abstract}
One of the important methods of creating positive motivation for students throughout the educational process is the application of a methodology called "uncompleted" situations. Usage of this technique have roots in human psychological behavior, according to Zeigarnik's effect, human remembers actions much better if they are incomplete, i.e. they were not resolved in a natural logical way. Such situations are recalled by our memory in similar circumstances.

This research work highlights and gives complete mathematical examples of typology of incomplete assigned tasks, which have different motivational potential. This paper places emphasis on the question pertaining to the moment of interruption during the execution of a define task. In common cases, this problem is solved by the teacher during the process of searching for a solution to the given task, or in process of proving a theorem. During the discussion about possible solutions the teacher tracks the "thought process" of his students, which directly react on formed situations and at the same time simultaneously open up to the subjective substance of it dynamically perception and conceptualization.

In the past it was revealed that there are definitive differences in the effect of "uncompleted" situations appearance amongst children, and it was established that effects are varied based on individual abilities and age.
\end{abstract}

\section{Introduction}

There are two known traditional approaches to educate students to effectively solve mathematical problems. Teacher poses appropriate questions (aka Socratic method), which lead to task solution (George Polya). Teacher constructs appropriate exercises and step-by-step solve them in classroom interactive sessions with students by providing hints (S. Shokhor-
Trotsky). In both cases as outlined above, the teacher has to ascertain when the process of giving hints should end and when the self study and research process should begin. How does one create a "uncompleted situation" point, which stimulates student's further research initiative, therefore avoiding the situations, where students are left on their own to solve the task, or on the contrary, are lead all the way to the final solution.

There is no definite answer to this question. It all depends on didactic goals and specific external circumstances. Based on the above-mentioned teacher applies different strategies to organize classroom research process. Such strategies correlate to different stages of incompleteness. However, in any event the interruption of the research process is only suitable in the cases, when students are completely occupied with the process, and encounter a situation which requires the application of a different perspective, which has no definitive path towards the expected outcome.

Using "Uncompleted" situations is based on psychological law. Take for example, you are close to completing a task but were unable to do so or you were interrupted by someone. You were unable to receive the result. What are some possible reactions to such a situation? They may include: dissatisfaction, affliction, disappointment and/or desire to continue work. In this case you would remember this situation for a long time. Russian psychologist Bluma Zeigarnik found in 1927 that people remember better that which is unfinished or incomplete. This reaction was referred to as the Zeigarnik's Effect. Later K. Levin, the American psychologist, explained this fact. Quasi-need arising when a person is intent on accomplishing a task but an interruption of such generates intense condition resulting in it not being satisfied.

All actions, completed and uncompleted, are linked to positive and negative emotions. "Uncompleted" actions, however, are more often affiliated with negative emotions than the positive. In order to minimize if not eliminate the negative emotions related to the uncompleted actions one must become empowered with the will to complete. This may be 
influenced by curiosity to maintain the task and/or the correlation between the genuine desires to achieve success with that of avoiding failure in conjunction with the feeling of responsibility.

Mathematics teachers can use "Uncompleted" situations for one or more of the following: additional stimulation for students' activity; an indicator of the level of cognitive motivation; strengthening of memory to retain important information.

Research on the links between action and result has revealed that "Uncompleted" teaching actions are more effective with certain subjects where their interdependence is logically clear. A typical example is an essential discipline, mathematics, where problemsolving is an essential medium for the teaching and learning process.

"Uncompleted" situation is a person's attitude to some task. The development of this attitude can be formed naturally or by external influences. Such attitude depends either on task, specific features of students or teaching strategy.

\section{Motivation}

Evolving educational system, fuelled by demands of today's society, is constantly raising bars for school graduates. Nowadays it's not enough to possess knowledge and skills, student must learn how to acquire those skills, and psychologically this means as a teacher we need to instill in students the desire to learn.

Building motivation to learn at school age is the central highlight of modern educational systems. Its relevance comes from constant improvement in the educational content, staging tasks to form self study skills in the students, and self education and research. Motivation plays an important function in education: it spurs student behavior, points it in a defined direction, organizes it, and gives it a personal essence and value.

Among basic tasks facing modern school teacher is the complex and important task of creating positive motivation towards learning among the students. It is clear, that without such motivation student learning process will be ineffective.

Motivational sphere contains several aspects: they are, need, motivation and goal. Let's examine the structure of motivational area in students as it relates to the process of education, i.e. what defines, inspires learning activity in a child.

Each human activity starts from needs and wants. Need, it's a focus of a child behavior, and emotional condition, which create a prerequisite for further activity. This need may be satisfied via different approaches. But without it, the child's activity is dormant, he doesn't' have motives and he is not ready to set goals.
Each child has a constant urge for new impressions which transforms into need for information. The teacher must lay the foundation to harness such a need, actualize it, and make it clear and transparent in minds of most students. The teacher must provide a transformation stage from needs to activity. In some cases learning needs are satisfied with acquisition of good marks, in others, with properly organized teaching process, they are satisfied via focusing students attention towards content of study material, methods and approaches used in the classroom activity.

Social tasks are acquired during the education period, they are need to involve in practices which have social impacts, paybacks to society, responsibilities to another persons, desire for self improvement.

Another important aspect in motivational sphere is motive, i.e. action directed towards the subject, internal psychological condition of the human, directly linked to properties of the subject, which receives the said action. Motive in education is student actions directed towards particular sides of the educational process. Actually, this includes student focus towards obtaining the knowledge, receiving good marks, getting parents praises and establishing desired relationships with their peers. In other words, student behavior during the educational process is always inspired by several motives.

Specifics of motive as a component of motivational sphere confined in the fact, that it is directly linked to the sense, personal significance of the actions, i.e. if motive, which is personal goal of the student, changes then the goal of entire educational activities drastically changes and vice versa.

Educational motives genesis is not a sufficient condition for an effective educational process, if the student, doesn't have the skills to place a goal in his/her studies. Goal is activity focus towards an intermediate result and usually represents a next step in achieving the subject of a need. In order to implement educational motive, for example, to acquire self study methods and skills, student require to place and achieve many intermediate goals, for example, learn to visualize certain results of their own study process, align the stages of today's study process with theirs, place targets for study activity and its self control. Besides, it is imperative that students learn how to be conscious about results of their actions and correlate them with motives of their study activity, in which such actions are included. Educational goal can remain the same (for example, acquiring self study skills) but sense of educational activity will change depending on its motive (for example, to become all-rounded person, achieve entry to the university and so on).

There is one more side to motivational sphere in the educational process, which is always mentioned in the 
schools, this side is interests towards the studies. Such interest is closely linked to the level offered by educational programs and in that sense it is an expression and manifestation of the other sides of motivational sphere such as motives and goals. Main feature of interest is emotional background, and connection to the child's emotional experiences. Of course, such feature is important, but it is not a main factor. It seems, that connection between child's interests with positive emotions have a value in the initial steps, because it sparks child's curiosity (for example in the new theme or new school subject), but to continue to maintain a stable level of interest, there is a need for an already formed, and proven educational activity, as well as abilities to self setting educational goals and their resolutions which are connected to the activity.

Any educational material cannot have positive motivational effect. Study material with informational content which appeals to the child's interests and newly emerging needs can have a positive impact. Contents of each lesson or classroom activity, must be deeply rooted and appeal to child's motivation. However, this content cannot consist of up-to-the-minute and easily forgettable moments or references to practical importance in the future endeavors (although teacher cannot avoid mentioning this fact), but such content must mainly be focused towards solving serious scientific problems, theoretical knowledge of phenomena, surrounding objects and the methods of acquiring such knowledge.

Study of motivation allows answers three main questions:

1. What force an individual to start performing certain actions? What are the personal needs, of the individual, which cause his/her activity?

2. Why people are determined in attempts to achieve their goals? What are characteristics of assertive individual who can stubbornly work towards the goal? (internal motivational factors).

3. What are the external conditions which induce individual motivations and allow him to keep his movement towards predefined goal? (external motivational factors).

If incorrect motivation is used, then subject shows affective reactions, such as refusal to do work, aggressive self-assertion, inactivity and apathy. Content of study materials plays important motivational role in educational process. One of the important methods in achieving high motivational level is introduction of uncompleted situations in the study process.

\section{3. “Uncompleted” situations}

The Table 1 illustrates typology of 6 categories of "uncompleted" situations which contain various motivational potential in a school math.

Table 1. "Uncompleted" situations

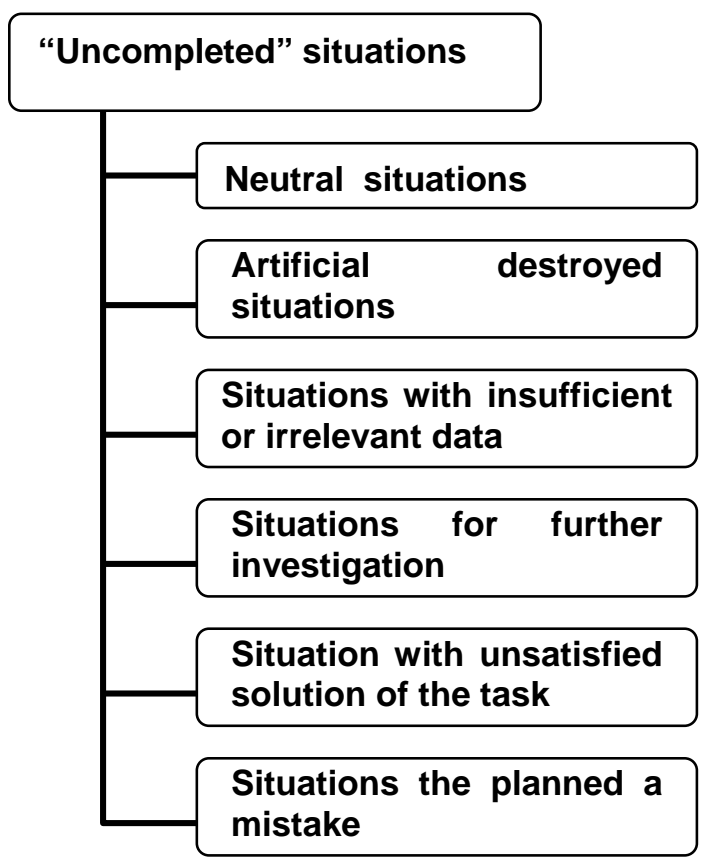

\subsection{Neutral situations}

Students' initial perception of a given problem before implementing standards based procedures.

\subsection{Artificially destroyed situations}

Interruption in the very early stages of the process, for example, after recording a data, in which the negative effect is minimal since the final goal is distant. Such situations, firstly, take place when the teacher comments quickly on the days' exercise. This obstructs the ability of students working independently, to think logically and analytically. As such this may negatively influence the students' score on the problems as compared to those completed alongside the teacher.

Secondly, if interrupted during the planning stage this interferes with the assimilation of the data, thus the process has to be repeated.

Nearing the completion of the task students attain additional motivation. Being fully immersed in the task and seeing the possibility of achieving their goal, any 
interruption may have adverse effects on its completion.

\subsection{Situations with insufficient or irrelevant data}

The teacher presents task to student with insufficient or irrelevant data. The student observes and revises it to an improved mathematical problem. We will check the hypotheses formulated by the students later.

Let us analyze the following task in which insufficient data is provided

\section{Task 1.}

$\mathrm{O}$ is the point of intersection of diagonals on the rectangle $\mathrm{ABCD}$ (Figure 1). The distance from $\mathrm{O}$ to the shorter side of the rectangle is $6 \mathrm{~cm}$ longer than to the longer side of the rectangle. Determine the lengths of each side.

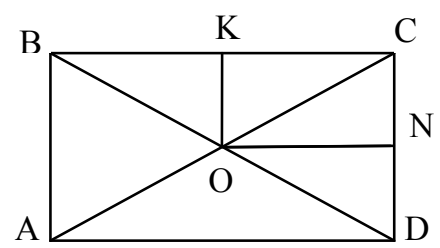

\section{Figure 1. Situations with insufficient or irrelevant data}

Data: In rectangle $\mathrm{ABCD}, \mathrm{OK}$ is $6 \mathrm{~cm}$ less than $\mathrm{ON}$. Determine the length of the sides of the rectangle.

Discussion of data

Teacher $(\mathrm{T})$ : What are we required to find in this problem?

Student (S): The length of the sides of the rectangle.

$\mathrm{T}$ : What follows from inequality $\mathrm{OK}<\mathrm{ON}$ by $6 \mathrm{~cm}$ ?

$\mathrm{S}: \mathrm{ON}-\mathrm{OK}=6$

$\mathrm{T}$ : If $\mathrm{OK}$ is equal to $\mathrm{x}$. What would $\mathrm{ON}$ be equal to?

S: $\mathrm{x}+6$

T: Can we use $x$ and $x+6$ to express side $A B$ ?

$\mathrm{S}$ : Yes, $\mathrm{OK}$ is half $\mathrm{AB}$, hence, $\mathrm{AB}=2 \mathrm{x}$.

$\mathrm{T}$ : What would be the length of $\mathrm{BC}$ ?

$\mathrm{S}$ : Since $\mathrm{ON}$ is half $\mathrm{BC}$, then $\mathrm{BC}=2(\mathrm{x}+6)$.

$\mathrm{T}$ : Did we use all the data given in the problem?

S: Yes.

$\mathrm{T}$ : Is this enough information for us to form the equation?

S: No.

$\mathrm{T}$ : What data is necessary to form the equation?

$\mathrm{S}$ : This given data should connect $\mathrm{AB}$ and $\mathrm{BC}$.
T: Bring examples of values which can show connection $\mathrm{AB}$ and $\mathrm{BC}$.

S: Perimeter, area, length of one side, diagonals, etc.

T: Choose one of these values. Set its value and solve the equation.

The student acknowledges the importance of additional information - perimeter, area, length of one side or diagonals - in order to complete the task.

\subsection{Situations for further investigation}

When discussing the attributes of the equality of triangles the teacher have the students formulate their own attributes. Their searches for possible attributes lead to the formulation of three "new" attributes: two angles and the side opposite to one of the angles, two sides and an angle opposite to one of the sides and three angles. The last attribute is easily denied by a counterexample. The correctness of the first two is not established until sometime in the future. Unfortunately, attempts to prove their attribute using the analogy of proven attributes were unsuccessful. As a result the students come to the realization that their knowledge is incomplete. This serves as a long-term motivator during the study of topics in the future.

\subsection{Situations with unsatisfied solution of the task}

Upon completing the task some students are not satisfied with the results claiming that there might be other possible solutions and so may visualize other techniques. The need for research of the solution is actualized and saved in students' motivational sphere.

\section{Task 2.}

For what value of a would the equation (a-1) $\mathrm{x}^{2}+2 \mathrm{x}-1=0$ have one root?

As customary the student may have been exposed to only the case when the discriminant of the equation equal zero. The teacher can utilize this opportunity by implying the presence of other solutions and suggests ways to determine the value of a needed for simplification of the equation. In particular, if $a=1$, we shall have the equation of the first degree with one solution, from which students perceptions of their solution is supported.

\subsection{Situations with planned mistake}

Such situations require immediate action on the part of the learner to do one or more of the following: correct data values in which contradictions exist to the rest of the data, properly diagnose and correct error in 
previous solution, along with the interpretation. These core skills are an essential way for the learner to develop critical thinking skills. Consequently, students, with or without their teacher, can acquire a better and deeper understanding of the specific concept by finding a sequence of actions that lead to their desirable goal.

\section{Task 3.}

A passenger plane is required to travel at an average speed of $1600 \mathrm{~km} / \mathrm{h}$ for an on time arrival. If the plane traveled the first half of the journey at $800 \mathrm{~km} / \mathrm{h}$, what speed, in $\mathrm{km} / \mathrm{h}$, is necessary to complete the entire journey on time?

A mean algorithm is used by the majority of students to solve this problem. Their solution to this problem, $2400 \mathrm{~km} / \mathrm{h}$, creates doubts about its reality, validity and computability, hence the quest for thorough investigation of the entire algorithmic process.

When the expected time of arrival was compared with the time taken for the first half of the journey, students were convinced that all planned time for the flight was spent on the first half of the journey. They concluded that the plane will not arrive on time regardless of the speed at which it travels the second half. Such contradiction invites students to revisit the content provided. This task would provide an insight into the reason for the contradiction, resulting in a restating of the question to read, "Is it possible for the plane to complete the entire journey by the given time?"

During specially designed conducted experiments we came to conclusion that interrupted action, having a form of "residual stress" of uncompleted action, appears only when, the other latent action, stronger in nature stops influence the first action.

In particular, the advantage of uncompleted task is not directly related to shock effect or additional emotional state, which occurs during interruption process of the given task and it's not linked to the person desire to remember an uncompleted task.

Moreover, if the current task is interrupted and new situation or task is carried out, then teachers can specifically trigger emotional "shake off" (for example cross referencing several situations or tasks), as a result the studied effect become less visible. For example, during classroom activity teacher tells the class that they are now going to look at more interesting example, and then introduces the task by using didactic materials, such as clear schema, computer graphics and so on). In such environment most students will not recall previous task, because they completely concentrate on new, more attractive subject.
Interrupting the task gets a special motivational sense, when a student (and not only a student) completely submerges in to the subject. Such moments called" points of maximum contact student with given task. During such contact moments with task (interruption moments) this task, usually, seems to be solvable and solution is achievable. Student already sees how to arrive to the final result and which methods to use to achieve the same and completely submerges in search of the solution with all his mind and personality, but he is not yet able to predict, how results will look like. Precisely in this case his work will not be demanded by superficial needs, formed as a result of initial intentions, but more likely by "natural" humans needs (ambitions, personal qualities, and active involvement of personal "Ego").

In practice, to find points of most close contact with the task is a difficult exercise. In general such problem can be solved by the teacher during collaborative search effort in the classroom, while explaining difficult problem or doing theorem proving. Teacher tracks students "thought process" during the classroom activities and discusses possible to solve given task and ensures that students do not "simply comment" already undertaken actions and operations, but directly react ever changing and evolving situation. By doing so students simultaneously open up subjective essence of such dynamically build up occasion and process of it realization. Interruption undertaken in precise moment of student being completely submerged in exercise solving and stumped with necessity of actualization of another.

Here are the basic most often user procedures for classroom mathematical task solving: generalization, unification, parameterization, introduction, transposition, specification, reduction, alteration, reconstruction.

The specified procedures, having a nature of creative mathematical thinking are reflected as separate entities or as a variety of combinations with each other. However relationships between subject of activity to any of such procedures is different from level to level. If at initial level application of any suitable method evaluated by student like an act of muse (guess = "insight"); then with moving on to more higher complex level, it usage become more cognizant, formed as a result of knowledgeable choice from the whole row of alternatives. Due to this the more choices student have, more comprehensive.

It is important to note that regardless of presented mathematical problems the greatest motivational impact achieved when the initiative comes from students in the form of educated guesses, suggestions and hypotheses during the process of applying different heuristics methods to the posed problem. 


\section{Conclusion}

As a result of the research, authors have discovered that Zeigarnik's effect can be expressed differently. This allowed to make following conclusions:

- Small children react more spontaneously to external stimuli while performing the task since they demonstrate the tendency to restart working once interrupted.

- Among children of one age it is possible to divide among three groups. They include children with: (a) high level of motivation; (b) medium level of motivation and (c) low concentration ability. Representatives of the second group have strong intention to finishing the task.

- Children who are motivated to succeed react differently to Zeigarnik's effect compare to children who are less inclined to do so because of the establishment of protective barriers.

- Interrupting a task, in which the student has little or no interest, is seldom associated with negative effect on the individual, as they easily forget uncompleted action.

- For some students, an interruption of the task does not sway their desire to complete; they readily do so without being directed by the teacher.

\section{Acknowledgements}

We would like to express our gratitude to Professor June George, Dr. Vladimir Korotov, Dr. Maria Byron, Dr. Susan Herbert, without their guidance and unwavering support, this research would not have been possible.

We would like to thank our friends Vanessa Perez, Oleg Smagin and Maria Valkova, without whose support this project would have been an uphill task. They provided assistance with document processing/formatting and notes in our research.

Special thanks should be given to all our colleagues who have helped us in many ways.

\section{References}

[1] V.V. Guzeev, Application in teaching effect of uncompleted action, Mathematics at school 1, Russia,1994, p.p.36-37.

[2] B.V. Zeigarnik, Memorization of the completed and uncompleted actions, Levin K. Dynamic psychology: Selected works, Russia, 2001.

[3] V.A. Ivannikov, Psychological mechanisms of strongwilled regulation, Moskow, URAO, 1998.
[4] V.G. Leontiev, Psychological mechanisms of motivation of teaching activity, Novosibirsk, NSPI, 1987.

[5] G. Polya, Mathematical Discovery on Understanding, Learning, and Teaching Problem Solving, Moskow, Science, 1976.

[6] M. Rodionov and S. Velmisova. Construction of Mathematical Problems by Students Themselves, Applications of Mathematics in Engineering and Economics: Proceedings of the 34th Conference on Applications of Mathematics in Engineering and Economics (AMEE '08); American Institute of Physics (Melville, NY 11747 USA) DOI:10.1063/1.3030789 AIP Conf. Proc. -- Issue Date: October 30, 2008 -- Volume 1067, pp. 221-228. 